\title{
Linx
}

Revue des linguistes de l'université Paris X Nanterre

9 | 1997

Émile Benveniste. Vingt ans après

\section{Benveniste et Saussure : l'instance de discours et la théorie du signe}

\section{Akatane Suenaga}

\section{(2) OpenEdition}

\section{Journals}

Édition électronique

URL : http://journals.openedition.org/linx/1011

DOI : 10.4000/linx.1011

ISSN : 2118-9692

\section{Éditeur}

Presses universitaires de Paris Nanterre

\section{Édition imprimée}

Date de publication : 1 avril 1997

Pagination : 123-128

ISSN : 0246-8743

\section{Référence électronique}

Akatane Suenaga, «Benveniste et Saussure : l'instance de discours et la théorie du signe », Linx [En ligne], 9 | 1997, mis en ligne le 05 juillet 2012, consulté le 01 mai 2019. URL : http:// journals.openedition.org/linx/1011; DOI : 10.4000/linx.1011 


\title{
Benveniste et Saussure : l'instance de discours et la théorie du signe
}

\author{
Akatane Suenaga
}

\section{Introduction}

$\mathrm{E}$ st-il nécessaire d'évoquer que Ferdinand de Saussure, en opposant la langue à la parole, a choisi la première Pour objet de sa science et a fondé, dit-on, la linguistique moderne? Il est aussi notoire que Emile Benveniste a appelé à "aller au-delà du point où Saussure s'est arrêté " (II, 219) ${ }^{1}$ et a été initiateur d'une théorie de la parole, qu'il a développée dans la perspective de ce qu'il appelle le discours. Concernant le problème du discours on a donc l'habitude d'opposer les deux linguistes.

Dans le présent exposé j'essaierai, en réexaminant la conception de la langue chez Saussure, de proposer quelques repères qui permettraient de voir une continuité entre elle et le discours tel que le présente Benveniste.

Dans mon analyse je retiendrai de Benveniste seulement deux problèmes qui sont liés à sa théorie de l'énonciation : 1) celui de la subjectivité, 2) celui du point de vue. Par "point de vue" j'entends celui qui détermine plus ou moins systématiquement le rapport langage/pensée chez le sujet et l'organisation des données de son expérience.

Dans cet esprit je vais examiner les textes où Benveniste traite les pronoms personnels et la subjectivité ${ }^{2}$, et chez Saussure quelques passages des sources manuscrites du Cours de linguistique générale ${ }^{3}$.

1 «La forme et le sens dans le langage» (1967), ch. XV dans Problèmes de linguistique générales II (tome I, 1966, tome II, 1974, Paris, Gallimard). Les références des citations de Benveniste sont données avec place dans PLG.

2 Il s'agit de «Nature des pronoms» (1956) et de «De la subjectivité dans le langage» (1958), respectivement ch. XX et ch. XXI dans PLG I. 


\section{Benveniste ou l'instance de discours}

Je commence par examiner le mot clé de la théorie de Benveniste développée sous le terme d'instance de discours, à savoir je, pronom personnel de la première personne du singulier. Quelles sont donc les propriétés de je par rapport aux autres signes?

Selon la définition de je donnée par Benveniste lui-même « je signifie "la personne qui énonce la présente instance de discours contenant je"» (I, 252). Il est aisé de voir là que cette définition de je permet de définir symétriquement $t u$ "comme l'“individu allocuté dans la présente instance de discours contenant l'instance linguistique $t u^{\prime \prime}$ » (I, 253). Je se trouve en effet au centre du phénomène qu'on appelle la coïncidence de l'acte d'énonciation et de l'action énoncée.

Il est à noter ici que cette propriété de je se traduit dans la particularité de sa référence : «ll n'y a pas de concept "je" englobant tous les je [...] au sens où il y a un concept "arbre" auquel se ramènent tous les emplois individuels de arbre. Le "je" ne dénomme donc aucune entité lexicale» (I, 261).

Or, je se distingue non seulement des noms porteurs d'une notion lexicale, mais aussi des formes qu'on présente traditionnellement dans son voisinage, on le sait, à savoir les pronoms personnels de la troisième personne que Benveniste qualifie de non-personne : «la non-personne est le seul mode d'énonciation possible pour les instances de discours qui ne doivent pas renvoyer à elles-mêmes, mais qui prédiquent le procès de n'importe qui ou n'importe quoi hormis l'instance même, ce n'importe qui ou n'importe quoi pouvant toujours être muni d'une référence objective» (I. 255-256).

Ainsi, sous cet angle benvénistien le monde, pour ainsi dire, se divise en deux en fonction de l'opposition personne/non-personne : d'un côté se trouve la sphère subjective articulée en je et $t u$, et de l'autre celle de la "troisième personne ». Cela signifie que c'est cet aspect pragmatique du discours qui joue un rôle essentiel dans la constitution de la subjectivité.

Alors, comment je se comporte-t-il dans le processus de cette constitution? Benveniste insiste ainsi sur la fonction, pour ainsi dire suidéclarative de je, essentielle dans le fondement de la subjectivité: «le fondement de la subjectivité est dans l'exercice de la langue. [ ... ] il n'y a pas d'autre témoignage objectif de l'identité du sujet que celui qu'il donne ainsi luimême sur lui-même» (I, 262). Mais cet auto-témoignage ne tient évidemment pas sans le partenaire qu'est $t u$, à qui je s'adresse: c'est cette condition de dialogue qui est constitutive de la personne, car «elle implique en réciprocité que je devient tu dans l'allocution de celui qui à son tour se désigne je » (I, 260).

La subjectivité, ainsi constituée, par la relation de je et tu que Benveniste appelle la polarité des personnes est intersubjectivité. Et là, il n'est pas difficile

3 Nous nous fondons sur le Cours de linguistique générale, édition critique par Rudolf Engler, fascicule 1-3. 1967-1968, fascicule 4, 1974, Wiesbaden, Harrassowitz. Les références des citations provenant de cet ouvrage sont indiquées par le numéro de fragment. 
de voir qu'un tel processus de construction subjective peut bien évoquer le problème du point de vue dans le sens que j'ai précisé plus haut. Rappelonsnous : le domaine de la troisième personne est un monde rempli de référence objective, appréhendé à partir du point de vue je, qui lui applique des prédicats et le saisit dans des concepts. Par contre je ne peut pas disposer d'un « concept "je" englobant tous les je». En effet, je n'est pas définissable, comme nous l'avons vu, sans recours à je lui-même : comme si je, en tant que point de vue, manquait de distance pour regarder l'objet qu'il est lui-même.

Ainsi le sujet parlant, en s'énonçant je, s'approprie le point de vue sans lequel il ne parviendrait pas à une prise de position vis-à-vis du monde, ce qui, selon Benveniste, signifie en même temps l'appropriation de la langue. Il s'agit, en l'occurrence, de l'organisation des indicateurs qui permet de déterminer toutes les relations spatio-temporelles: "Ce sont les indicateurs de la deixis, démonstratifs, adverbes, adjectifs, qui organisent les relations spatiales et temporelles autour du "sujet" pris comme repère: "ceci, ici, maintenant", et leurs nombreuses corrélations "cela, hier, l'an dernier, demain" etc » (I, 262). Le système déictique des indicateurs, organisé autour du noyau je, constitue ainsi la grille spatio-temporelle sur le fondement de laquelle l'expérience est donnée au sujet qui, à partir de là, applique des prédicats au monde.

Récapitulons: selon Benveniste, le seul geste de s'énoncer je, acte essentiellement pragmatique, fraye la voie vers: 1) la constitution de la subjectivité dans la relation dialectique avec $t u, 2)$ la concomitance de l'appropriation de la langue même et de l'établissement du point de vue qui détermine la façon subjective d'articuler et de construire les relations avec le monde. Je propose la synthèse suivante de ces deux points : étant donné que la subjectivité se constitue dans l'échangeabilité de je et $t u$, et que je est, comme on $\mathrm{a} v \mathrm{vu}$, le point de vue à partir duquel le sujet prédique le monde, le dialogue, entre je comme locuteur et $t u$ comme allocuteur, n'est autre que l'échange de la position subjective vis-à-vis de leur partenaire et du monde. Autant dire que la constitution de la subjectivité implique une intériorisation de la polarité de je et $t u$ dans chacun des sujets parlants : pour ainsi dire, à l'intérieur du locuteur je il $\mathrm{y}$ a donc un $t u$ qui l'écoute ${ }^{4}$ : à preuve, on n'arriverait jamais à parler sans entendre sa propre voix.

\section{Saussure ou la théorie du signe}

Alors, qu'en est-il donc du problème de la subjectivité et de celui du point de vue dans la réflexion de Saussure?

Commençons par le point de vue. A ce propos Saussure est formel : la langue est un point de vue tellement imposée au sujet parlant que celui-ci n'en

${ }^{4}$ Cf. «Le "monologue" est un dialogue intériorisé, formulé en "langage intérieur", entre un moi locuteur et un moi écouteur. Parfois le moi locuteur est seul à parler; le moi écouteur reste néanmoins présent ; sa présence est nécessaire et suffisante pour rendre signifiante l'énonciation du moi locuteur » : Benveniste, «L'appareil formel de l'énonciation » (1970) (II, ch. V, 85-86). 
est même pas conscient. Nous citons dans la première leçon du deuxième cours de linguistique générale, donnée en novembre $1908^{5}:$ : la langue [...] nous paraît tout près de notre main : peut-être est-elle trop près ([...] verre de la lunette par lequel et au travers duquel nous saisissons les autres objets) " (frag. 1766).

D'ailleurs, cette idée de langue comme point de vue est corrélative de la fameuse conception de la langue comme système de différences négatives. Mais je ne développerai pas cela, je remarque seulement quelques conséquences de cette conception : 1) ce n'est pas l'objet correspondant qui détermine la valeur d'un signe, mais sa coexistence avec les autres signes, autrement dit le découpage entre eux ; 2) les différences négatives, par définition, ne parvenant pas comme telle à la conscience du sujet, celui-ci n'a qu'à se soumettre à la distribution des valeurs que lui impose sa langue. D'où la langue comme point de vue subi par le sujet.

Alors se pose la question suivante : comment la segmentation particulière de la langue, autrement dit l'association déterminée d'un signifiant à son signifié est-elle décidée ? Il s'agit du problème de la subjectivité chez Saussure. Qu'est-ce à dire?

Reprenons la première leçon du deuxième cours. Saussure propose là, comme raisons de la difficulté de déterminer l'objet de la linguistique, plusieurs dualités de la langue. Je vais examiner les trois premières: «les syllabes qu'on articule ne sont-elles pas dans le son, dans ce que perçoit l'oreille? Oui, mais les sons ne seraient pas existants sans les organes vocaux. Donc, si on voulait réduire la langue au son, on ne pourra la détacher des articulations buccales : et réciproquement, on ne peut même pas définir les mouvements de l'organisme vocal en faisant abstraction de l'impression acoustique. [...] Ainsi dans le phonème $k$ : deux côtés qui ne font qu'un par leur correspondance » (frag. 134, 136, 135). Voilà la dualité articulatoire/ acoustique du son linguistique.

Ensuite la deuxième dualité : «le son vocal n'est un mot que dans la mesure exacte, constante, qu'il lui est attaché un sens. Cette correspondance se vérifie à chaque pas de la linguistique à tel point qu'on ne peut dire ce que c'est qu'une forme sans prendre à la fois le son et le sens » (frag. 139). Voilà la deuxième dualité, celle du signe comme ensemble d'un son et d'un sens.

Saussure passe à présent à la troisième: "cette unité complexe doit trouver sa sphère au moins dans deux individus: donc troisième unité complexe $\{-x$. Le passage de la bouche d'un monsieur A à l'oreille d'un monsieur B et réciproquement sera toute la vie de la langue. [ ... ] La langue est faite pour communiquer avec ses semblables. Enfin ce n'est que par la vie sociale que la langue reçoit sa consécrations » (frag.142). Voilà la troisième dualité : l'intersubjectivité du langage.

5 Les dates des trois cours de linguistique générale professés à l'Université de Genève sont : I, 1907, II, 1908-1909, III, 1910-1911. 
Nous remarquons d'abord la relation que Saussure définit entre la deuxième et la troisième dualités : il s'agit ici évidemment de l'interdépendance entre la langue et la parole. La langue sert à la parole qui dépend d'elle. Mais il est également vrai que la langue a son tour besoin de la parole pour qu'elle reçoive «sa consécrations ». Le signe comme double unité représentant ici la langue, la consécration de celle-ci par la vie sociale, à savoir par le processus de parole, signifie bien celle du signe, i. e. celle de l'association d'un signifiant à son signifié correspondant.

Nous remarquons aussi que le processus de parole est présenté ici comme " passage de la bouche d'un monsieur A à l'oreille d'un monsieur B et réciproquement...». Cette description nous renvoie au schéma de communication proposé deux ans et demi plus tard dans le troisième et dernier cours, que Saussure appelle "circuit de la parole" :
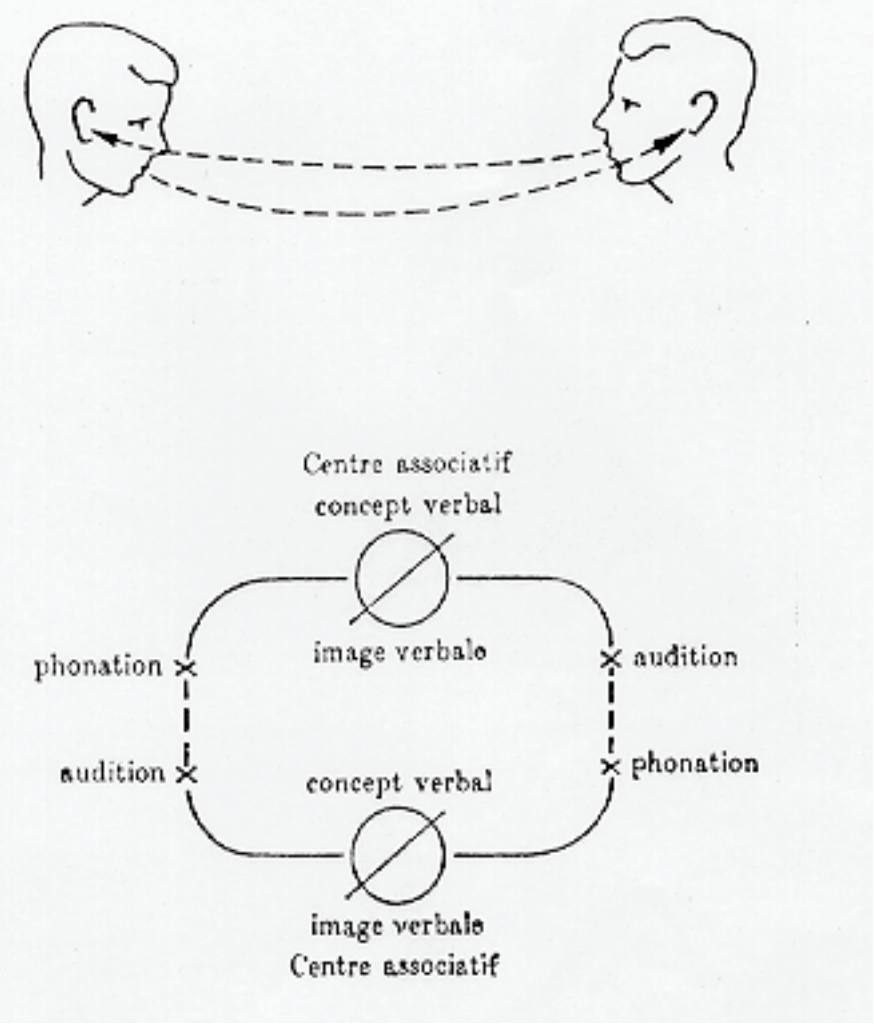

(frag. 194-196, 198)

L'intention qu'a Saussure proposant ce schéma est de dégager du langage comme ensemble hétéroclite la langue comme objet de sa linguistique. Saussure essaie de localiser le siège de la langue parmi différentes strates du circuit qu'il divise de plusieurs façons, soit en physique/ physiologique/ psychique, soit en exécutive/ réceptive etc. 


\section{Akatare Suenaga}

En appelant la formation du dépôt de la langue dans l'individu "capitalisation" et "cristallisation sociale", Saussure s'interroge comme suit: "Quelle partie du circuit peut donner lieu à cette capitalisation, cristallisation sociale ? Ce n'est pas une partie quelconque : ce n'est pas la partie physique. Ainsi nous sommes frappés d'une langue étrangère que nous ne connaissons pas, mais nous ne sommes pas dans le fait social de la langue » (frag. 223, 226). Le son d'une langue inconnue ne constitue pas "le fait social de la langue" parce que, pour Saussure, celui-ci implique que le son perçu est appréhendé grâce à la coordination qui le rend compréhensible. Saussure prend donc pour lieu de la langue «la partie réceptive et coordinative (qui est sociale) » : «ce qui forme un dépôt chez les différents individus, [... ] Ce sont ces milliers d'images verbales associées chez les individus à autant de concepts placés en regard» (frag. 229, 232). Saussure localise ainsi la langue dans la partie où le lien qui associe le signifiant au signifié se détermine et se réétablit au cours du langage: «Dans le centre associatif, purement psychique, sont mis en contact un concept verbal et une image acoustique » (frag. 198).

Cette mise en contact des deux faces du signe implique que la configuration des valeurs des signes dans la langue reçoit la consécration sociale en plein acte d'énonciation, i. e. dans l'instance de discours. Le circuit de la parole représente donc le processus où la polarité des personnes je et $t u$ s'intériorise dans la langue. Pour Saussure, la langue n'est rien d'autre qu'une intériorisation de la relation sociale de la parole dont l'unité élémentaire est celle des deux sujets parlants je et $t u$ : «On peut dire qu'en prenant un individu nous aurons dans le seul exemplaire l'image de ce qu'est la langue dans la masse sociale. Si nous pouvions examiner le dépôt des images verbales dans un individu, conservées, placées dans un certain ordre et classement, nous verrions là le lien social qui constitue la langue) (frag. 232-234).

Pour conclure, la conception saussurienne de la langue est ainsi profondément traversée par une inspiration quasi-benvénistienne de l'énonciation, mais avant la lettre, comme si pour Saussure, jusque dans un simple son linguistique, résonnait la voix double des deux sujets je et $t u$. Rappelons la première dualité que j'ai évoqué: «les sons ne seraient pas existants sans les organes vocaux. [ ... ] réciproquement, on ne peut même pas définir les mouvements de l'organisme vocal en faisant abstraction de l'impression acoustique. Ainsi dans le phonème $k$ : deux côtés qui ne font qu'un par leur correspondance ». 